\title{
Massive pulmonary embolism with ST elevation in leads V1- V3 and successful thrombolysis with tenecteplase
}

\section{G Livaditis, M Paraschos, K Dimopoulos}

Heart 2004;90:e41 (http://www.heartjnl.com/cgi/content/full/90/7/e41). doi: 10.1136/hrt.2004.036335

A 42 year old woman was referred to the emergency department from the radiology department after having a syncopal episode during a triplex examination of the lower extremities for acute painful right leg swelling five hours earlier that morning. She had no significant medical history, smoked 3-5 cigarettes a day, and had been taking contraceptive medication for menorrhagia for the preceding three months. On presentation she was cyanotic, dyspnoeic, and haemodynamically unstable. ECG showed sinus tachycardia of 120 beats/min with ST elevations from V1 through V3 mimicking anteroseptal acute myocardial infarction. Ten minutes after presentation she was thrombolysed with $80 \mathrm{mg}$ tenecteplase leading to ST elevation resolution and remarkable haemodynamic recovery after 20 minutes. This case shows how pulmonary embolism can mimic anteroseptal acute myocardial infarction on ECG and the life saving results from rapid thrombolysis with tenecteplase.

$\mathrm{P}$ ulmonary embolism (PE) is most often a complication of venous thrombosis, usually of the lower extremities and the pelvis. Massive PE is life threatening if left untreated, with rapid progression and deterioration. ECG abnormalities can be seen in $70-80 \%$ of patients, varying from the typical S1Q3T3 pattern to no specific changes. Very rarely ECG mimics anteroseptal infraction. We present a case of a patient who was successfully thrombolysed with tenecteplase. Despite the ECG pattern suggestive of acute myocardial infarction (AMI), her coronary arteries on coronography were normal. We also reiterate the usefulness of the echocardiogram in distinguishing AMI from PE in such cases. From a literature review we found very few reports of PE presenting with such an ECG pattern. This is the first report of successful thrombolysis with tenecteplase that resulted in early thrombus resolution and in haemodynamic stability in about 30 minutes.

\section{CASE REPORT}

A 42 year old woman presented to the vascular surgery department with acute, painful, right leg swelling beginning five hours earlier that morning after waking. Her weight was $80 \mathrm{~kg}$ and height $1.65 \mathrm{~m}$, she had smoked 3-5 cigarettes a day for the preceding 10 years and had been taking contraceptive medication for menorrhagia for the preceding three months. She had had an abortion four months previously. She had no significant medical history and was healthy up to the preceding five hours.

She was referred to the radiology department for examination of the lower extremities, which showed ileofemoral vein thrombosis. During the examination she noted sudden abdominal pain, became short of breath, and had a syncopal episode for $20 \mathrm{~s}$. She was then transferred to the cardiology emergency department for resuscitation.
On admission to the emergency department she had regained consciousness and was confused. Her blood pressure was $60 / 30 \mathrm{~mm} \mathrm{Hg}$, pulse 120 beats/min, respiratory rate 30 breaths $/ \mathrm{min}$, and oxygen saturation $81 \%$ in room air.

Physical examination found an overweight woman, disoriented to place, and confused. Her pupils were equally round and reactive to light, there were no signs of external injury, her chest wall was clear bilaterally on auscultation, heart rate was regular at 120 beats/min with normal first and second heart sounds, and with no clear mark of additional sounds due to the respiratory distress. The right leg was swollen and tender but well perfused and the abdomen was painful with guarding and rebound.

The initial 12 lead ECG showed a sinus tachycardia of 120 beats/min with ST elevations in V1 to V3, S wave and ST depression in DI, ST depression in D2, ST elevation in aVR, ST depression in aVL, Q wave and ST elevation in VI, ST elevation in V2 and V3, and ST depression in V5 and 6 (fig 1)

Arterial blood was drawn for blood gas evaluation of troponin I, glucose, blood urea nitrogen, creatinine, potassium, sodium, aspartate aminotransferase, alanine aminotransferase, $\gamma$-glutamyltransferase, creatine kinase, creatine kinase $\mathrm{MB}$ fraction, lactate dehydrogenase, prothrombin time, activated partial thromboplastin time, international normalised ratio (INR), fibrin degradation products, Ddimers, and fibrinogen. An intravenous line was placed and the patient was heparinised with $5000 \mathrm{U}$ of unfractionated heparin at $1000 \mathrm{U} / \mathrm{h}$. She was given $500 \mathrm{mg}$ of aspirin and $50 \%$ oxygen delivered through a Venturi mask at a rate of $10 \mathrm{l} / \mathrm{min}$. She was monitored continuously by ECG telemetry.

Cardiac ultrasound done in the emergency department showed a normally contracting left ventricle, a distended right ventricle with free wall hypokinesia, and displacement of the interventricular septum towards the left ventricle.

Spiral computed tomography (CT) was planned but because of haemodynamic instability was postponed for later that day. An $8000 \mathrm{U}$ bolus of tenecteplase was delivered over $10 \mathrm{~s}$ to a peripheral vein and the patient was transferred to the intensive care unit. The patient was haemodynamically stable 20 minutes after the tenecteplase infusion.

Figure 2 shows the ECG one hour after the thrombolytic infusion. The ST elevations in leads aVR and V1 to V3 resolved and the S wave in D1 and ST depressions in D1, D2, aVL, V5, and V6 disappeared.

The cardiac ultrasound later that day showed a normal left ventricle with an ejection fraction of $65 \%$ and a less distended right ventricle. There was still diffuse hypokinesia but the interventricular septum position and motion were normal.

The patient was well, with no complaints except for pain in the right leg, which was in a recumbent position. There was no cardiac enzyme activity that day or in blood analysed later.

Abbreviations: $\mathrm{AMI}$, acute myocardial infarction; $\mathrm{CT}$, computed tomography; $\mathrm{PE}$, pulmonary embolism; $\mathrm{RBBB}$, right bundle branch block; IVC, inferior vena cava 


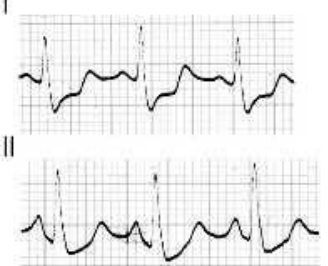

III

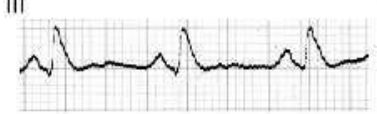

V1

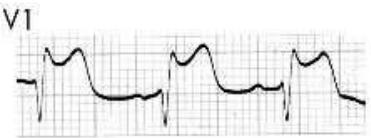

V2

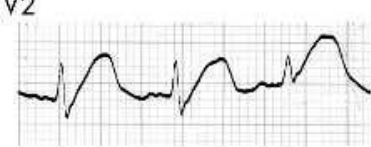

V3

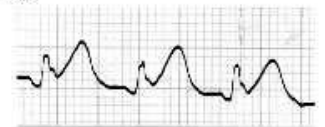

AVR

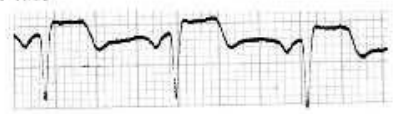

$\mathrm{AVL}$

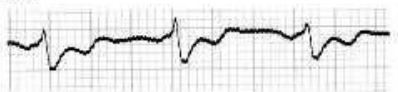

AVF

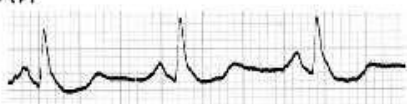

V4

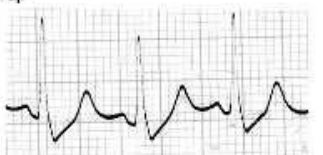

V5

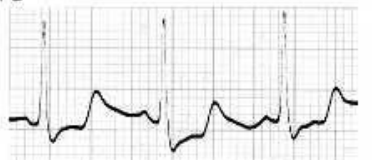

V6

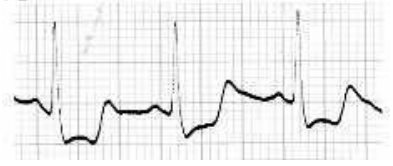

Figure 1 Initial ECG on admission.

The contraceptive medication was stopped on admission and, as she was given heparin and warfarin anticoagulation which was expected to begin two to three days after cessation of the hormone treatment, we decided to initiate monthly triptorelin administration. Hormone replacement was successful and she had no menstrual bleeding.

On coronography, the coronary arteries were normal and a left ventriculogram showed no wall motion abnormalities and an ejection fraction of $60 \%$.

Lung spiral CT and lower abdominal CT were done on the fourth day and were negative for PE. However, they showed ileofemoral thrombosis and a $7 \mathrm{~cm}$ long by $2.5 \mathrm{~cm}$ wide floating thrombus in the inferior vena cava (IVC) (fig 3) and enlargement of the uterus with uneven texture measuring $7.5 \times 7 \times 10 \mathrm{~cm}$.

A retractable IVC filter was placed while the patient was still being anticoagulated. A second lower extremity triplex examination was done on the 10th day still showing some

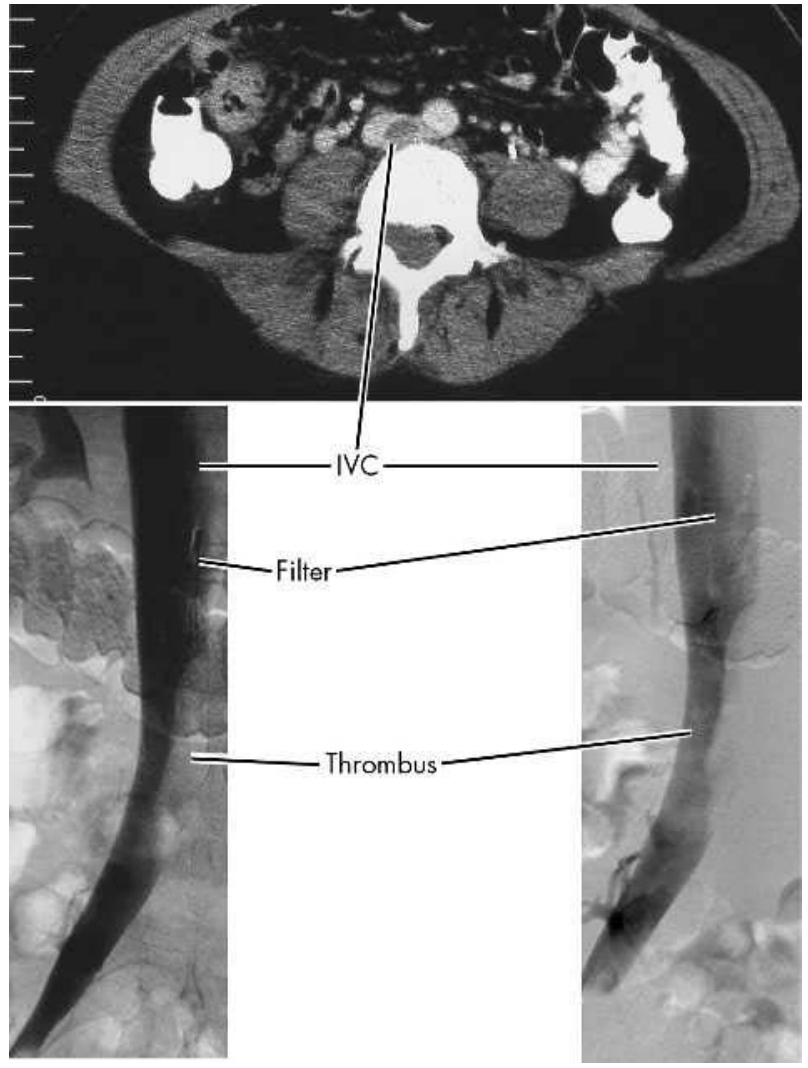

Figure 3 Computed tomography showing the inferior vena cava (IVC) thrombus and retractable filter in place.

extent of ileofemoral thrombosis. A ventilation perfusion lung scan was negative in the 15 th day.

The patient left after 20 days of hospitalisation in good condition, taking warfarin with an INR of 3 to 3.5, and triptorelin monthly till the end of the anticoagulation. She was instructed to be evaluated by a specialised obstetrics gynaecology centre and to have a thrombophilia assessment one month after the cessation of anticoagulation by measurement of proteins S and C, antithrombin III, and lupus anticoagulant.

We continued the follow up for a year. PE or other thrombosis did not recur. The patient is still taking warfarin anticoagulation and the IVC filter is still in place without signs of occlusion. Her uterus will be removed in the near future, after which the retractable IVC filter will be removed.

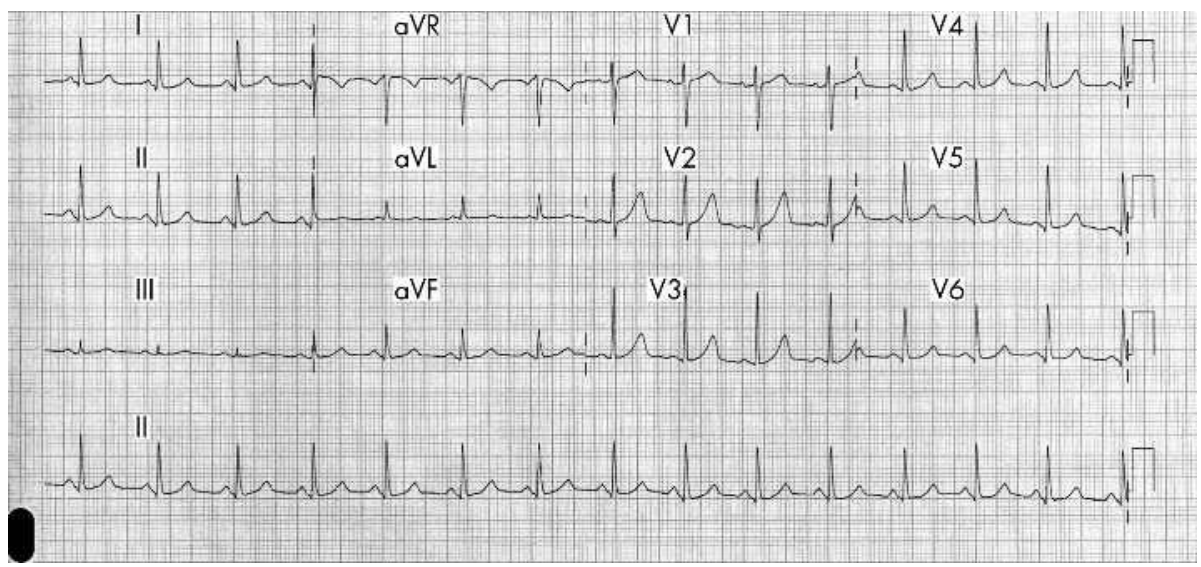

Figure 2 ECG one hour after infusion of tenecteplase. 


\section{DISCUSSION}

$\mathrm{PE}$ is the implantation of material into branches of the pulmonary arterial bed. This material usually consists of clots dislocated from peripheral veins but can also be neoplastic cells, fat emboli, amniotic fluid, inspirations from the air, pieces of catheters and other diagnostic devices, or exogenic material when delivered in the veins. Most commonly the clots migrate from deep veins of the pelvis and the lower extremities. The classical triad of Virchow is the main cause of such clots (endothelial injury, stasis, hypercoagulability).

When the material is embolised in the periphery of the pulmonary arterial tree the PE is subclinical and the diagnosis is difficult and most often missed. Massive PE caused by disruption of flow in more proximal positions is life threatening and the haemodynamic compromise depends on the dimension of the clot, the site, and the rapidity of the occlusion. Massive PE consists of shock or hypotension (defined as systolic blood pressure $<90 \mathrm{~mm} \mathrm{Hg}$ or a pressure drop of $\geqslant 40 \mathrm{~mm} \mathrm{Hg}$ for $>15$ minutes if not caused by new onset arrhythmia, hypovolaemia, or sepsis). The increased right ventricular afterload leads to increased right ventricular myocardial work and oxygen consumption. The cardiac index falls despite adequate blood pressure, constant or increasing right ventricular preload, and constant contractility. As the systemic pressure ultimately falls and the right ventricular pressure increases, the pressure gradient between the aorta and the right ventricle narrows leading to decreased driving pressure mainly in the right coronary artery. The combination of hypoperfusion and increased demand on the right ventricle leads to severe ischaemia and dysfunction, feeding a vicious circle leading to shock and high death rates. Mittal and colleagues ${ }^{1}$ described a case of submassive PE with isolated infarction of the right ventricular anterior wall presenting with sustained ventricular tachycardia. Cardiac ischaemia, however, does not entirely explain the deterioration of left ventricular output, which is also likely to be the result of the pericardial constraint in the face of right ventricular dilatation, as well as to a leftward shift of the interventricular septum, which reduces and deforms the left ventricle. Mortality after the initial thromboembolic event varies with the extent of PE and the patient's pre-existing cardiopulmonary status. When the initial embolic event is fatal, death often occurs within one to two hours. The likelihood of a recurrent embolus in an untreated patient is about $50 \%$ and as many as half of these recurrences may be fatal. Anticoagulation reduces the rate of recurrence to about $5 \%$; only about $20 \%$ of these are fatal.

The ECG is often abnormal in PE but the findings are insensitive and non-specific. ECG findings are usually nonspecific and almost $33 \%$ of patients have a normal ECG. The most common findings are sinus tachycardia and nonspecific ST segment and $\mathrm{T}$ wave changes. Changes that strongly suggest PE indicate strain on the right side of the heart; these changes include $\mathrm{T}$ wave inversion in precordial leads Vl through V4, transient right bundle branch block (RBBB), new right or left deviation of the QRS axis, sudden onset of atrial fibrillation or other atrial arrhythmias, and ECG signs of right ventricular hypertrophy or right atrial enlargement. The S1Q3T3 pattern also suggests PE. This pattern of right sided heart strain is usually accompanied by $\mathrm{T}$ wave inversion in the precordial leads.

$\mathrm{Chou}^{23}$ suggests that the following findings are typical: (1) an SIQ3 or SIQ3T3 pattern; (2) a rightward QRS axis shift; (3) transient, complete, or incomplete RBBB; (4) $\mathrm{T}$ wave inversion in the right precordial leads; and (5) sinus tachycardia. Other more rare findings are (a) left displacement of the transitional zone; (b) left axis; (c) QR in Vl; (d) $\mathrm{R}>\mathrm{S}$ in VI; (e) ST elevation in lead III; (f) ST changes in the right precordial leads; (g) atrial arrhythmias; and (h) first degree atrioventricular block.

Ferrari and colleagues ${ }^{4}$ studied 80 patients hospitalised in a coronary care unit with acute PE, all of whom underwent pulmonary angiography, and found negative T waves in VI-V4 in $68 \%$, the SIQ3T3 pattern in 50\%, peripheral low voltage in $29 \%$, sinus tachycardia in $26 \%$, complete or incomplete RBBB in $22 \%$, pulmonary $\mathrm{P}$ wave in $5 \%$, and a normal ECG in $9 \%$. In massive PE (determined angiographically), an anterior ischaemic pattern was noted in $85 \%$ of patients versus $19 \%$ in nonmassive PE. For massive PE anterior T wave inversions had a sensitivity of $85 \%$, a specificity of $81 \%$, a positive predictive value of $93 \%$, and a negative predictive value of $65 \%$.

Sreeram and colleagues ${ }^{5}$ reviewed the value of the 12 lead ECG at hospital admission in the diagnosis of PE. Acute ECG features were analysed in 49 patients with proven PE and echocardiographically proven acute right sided pressure increase. PE was considered probable from the presence of three or more of the following abnormalities: (1) (in)complete RBBB associated with ST segment elevation and positive $\mathrm{T}$ wave in lead Vl; (2) S waves in lead I and aVL $>1.5 \mathrm{~mm}$; (3) a shift in the transition zone in the precordial leads to V5; (4) Q waves in leads III and aVF but not in lead II; (5) right or indeterminate axis deviation; (6) a low voltage QRS complex $<5 \mathrm{~mm}$ in the limb leads; and (7) $\mathrm{T}$ wave inversion in leads III and aVF or leads V2-V4.

Falterman and colleagues ${ }^{6}$ reported a case of PE with ST segment elevation in leads VI-V4 in a patient who died despite resuscitative efforts. That patient had typical ECG findings-an SIQ3 pattern, right axis deviation, and incomplete RBBB-and other findings not considered typical-ST elevation in leads Vl-V4 and III mimicking anteroseptal infarction.

In our case the ST elevation was confined to the anteroseptal wall, the axis was normal, there were $\mathrm{S}$ waves in leads I and aVL $>1.5 \mathrm{~mm}$, the transaction zone was in V3, and there was ST depression in V6.

Although the ECG in our case was not typical of PE and could not differentiate AMI from PE, other examinations were very helpful. Cardiac ultrasound is a readily available bedside test that can be done in the emergency department at the initial presentation but rarely provides direct visualisation of PE. Instead, the diagnosis must be inferred from the observation of right ventricular dysfunction. Studies of patients with documented PE have shown that $80 \%$ of patients have imaging or Doppler abnormalities of right ventricular size or function that may suggest PE. Echocardiographic signs consistent with PE include tricuspid regurgitation, right ventricular dilatation, regional right ventricular wall motion abnormalities, paradoxical septal wall motion, and widening of the pulmonary artery diameter. In our case the examination was helpful in the differential diagnosis of PE and anteroseptal AMI.

Ultrafast imaging (spiral computed tomography) after contrast injection permits excellent visualisation of the pulmonary arteries and direct visualisation of pulmonary arterial clots in larger vessels. The sensitivity and specificity of spiral CT appear to be quite good for a proximal clot in the main, lobular, or segmental pulmonary arteries (sensitivity $94 \%$, specificity $94 \%$, positive predictive value $93 \%$, negative predictive value $95 \%$ ). Spiral CT has limitations, including poor visualisation of the peripheral areas of the upper and lower lobes, possible inadequate scanning of horizontally oriented vessels in the right middle lobe and misidentifying smaller lymph nodes as emboli. In addition, it requires the use of contrast dye and interpretation by an experienced radiologist. In the case of our patient the examination was done four days after the infusion so no clots in the major branches were observed. Nevertheless, the examination was 
helpful in identifying the IVC thrombus and to plan the implantation of the IVC filter.

D-dimer and other laboratory studies, although generally not helpful in establishing the diagnosis of PE, are potentially useful screening tools for PE. D-dimer indicates ongoing fibrinolysis. A quantitative D-dimer concentration of $<500 \mathrm{fg} / \mathrm{l}$ ruled out PE in $98-100 \%$ of cases. However, since any process that activates the coagulation cascade and fibrinolytic system may increase concentrations, the test is non-specific but its remarkable sensitivity gives an excellent negative predictive value.

In PE the pattern of ST elevation in anteroseptal wall is extremely rare. Cardiac ultrasound can contribute to the differential diagnosis from anteroseptal infarction in such cases. Early thrombolysis may be life saving and if the patient is very haemodynamically unstable, reperfusion with tenecteplase can lead to a rapid recovery. The evaluation of the patient must continue to determine the cause of PE and to prevent recurrence if possible. In selected cases the implantation of an IVC filter is needed. A retractable filter is a logical approach when the cause of the PE is expected to resolve in time.
Authors' affiliations

I G Livaditis, M Paraschos, K Dimopoulos, 1 st Cardiology Department, Red Cross Hospital, Athens, Greece

Correspondence to: Dr loannis G Livaditis, Red Cross Hospital, Athens 11635, Greece; livaditisj@yahoo.com

Accepted 17 March 2004

\section{REFERENCES}

1 Mittal SR, Arora H. Pulmonary embolism with isolated right ventricular infarction. Indian Heart J 2001;53:218-20.

2 Chou T. Electrocardiography in clinical practice, 2 nd ed. Orlando: Grune Straton, 1986:309-17.

3 Chou T, Knilans TK. Electrocardiography in clinical practice adult and pediatric, 4th ed. Philadelphia: Saunders, 1996:167-70.

4 Ferrari E, Imbert A, Chevalier T, et al. The ECG in pulmonary embolism: predictive value of negative t waves in precordial leads: 80 case reports. Chest 1997; 111:537-43.

5 Sreeram N Cheriex EC, Smeets JLRM, et al. Value of the 12-lead electrocardiogram at hospital admission in the diagnosis of pulmonary embolism. Am J Cardiol 1994;73:298-303.

6 Falterman TJ, Martinez JA, Daberkow D, et al. Pulmonary embolism with ST segment elevation in leads V1 to V4: case report and review of the literature regarding electrocardiographic changes in acute pulmonary embolism. $J$ Emerg Med 2001;3:255. 